\title{
ERRORES CONCEPTUALES EN GEOLOGÍA: LOS CONCEPTOS ISOTROPÍA-ANISOTROPÍA Y PROPIEDAD ESCALAR - PROPIEDAD VECTORIAL
}

\author{
GALLEGOS, J.A. \\ Departamento de Didáctica de las Ciencias Experimentales. Úniversidad de Granada.
}

\begin{abstract}
SUMMARY
Herein an analysis is offered of the conception that first cycle university students have about the terms IsotropyAnisotropy and Scale properties-Vectorial properties, that have been learnt only within the school limits. It seems, therefore, that if there are conceptual mistakes, they must be blamed on the Teaching-Learning system, and not on the previous ideas students might have.
\end{abstract}

\section{INTRODUCCIÓN}

Uno de los aspectos de mayor actualidad en la Didáctica de las Ciencias es la exploración de los conocimientos previos de los alumnos, para poder llevar a cabo, sobre ellos o a cambio de ellos, la construcción del estado actual del conocimiento científico en cualquiera de los campos de su ámbito. En este terreno, entre otros, se mueve también mi investigación didáctica.

Pero más de una vez, los resultados han sido tales que permitían sacar un abanico de conclusiones, no todas relacionadas con el presupuesto (implícito o explícito de este tipo de sondeos), de que es la mente del estudiante la que ha elaborado sus esquemas de acuerdo cort su propia percepción del mundo que le rodea. Ésa fue la razón de que enfocara mi atención hacia el análisis de conceptos que no pertenezcan, en modo alguno, al mundo normal de los conocimientos vulgares, sino que sean exclusivamente fruto del aprendizaje en el aula. Pretendía, así, delimitar hasta qué punto puede ser causante de errores el propio proceso de enseñanza-aprendizaje, mal enfocado. Los conceptos de Isotropía-Anisotropía y Propiedad Escalar - Propiedad Vectorial me parecieron muy adecuados: porque cumplen el requisito anterior (no pertenecen al lenguaje vulgar en absoluto), porque se detecta un proceso tautológico en los enunciados emitidos por los alumnos y porque se repiten varias veces en la enseñanza de la Geología (lo que permite hacer un seguimiento de la evolución de su compresión a lo largo de varios cursos).
En esta nota sólo se analizarán las conclusiones obtenidas con Ios alumnos de Primer Ciclo de Universidad que volvían a estudiar Geología (incluyendo estos conceptos) por primera vez a nivel universitario, y se iratará de detectar si la equívoca idea que tienen se debe a un mal aprendizaje o a una mala enseñanza de los mismos. Para ello se partió de las respuestas a una encuesta en que se pedían las cuatro definiciones, se analizaron los resultados, y se trató de encontrar posibles conexiones con las explicaciones impartidas por los profesores o con los textos de Ciencias Naturales más usados en el Bachillerato. Excepcionalmente, se encontraron algunos alumnos que ya habían cursado las asignaturas de Geología o Mineralogía en cursos universitarios (por ser repetidores o por haber cambiado de licenciatura) y los errores eran exactamente los mismos; dado su escaso número, no se puede decidir si también a este nivel siguen dándose confusiones en la enseñanza de estos conceptos o pueden ser pervivencias anteriores porque no se ha vuelto a tratar este aspecto.

\section{LA DEFINICIÓN DE LOS CONCEPTOS Y SU JUSTIFICACIÓN}

Antes de iniciar un estudio de este tipo parece imprescindible establecer cuáles son los conceptos de referencia y justificar por qué se entienden de esa forma; sólo así se 
podrá llevar a cabo el análisis posterior. Por eso se definen los conceptos y se justifican esas definiciones a continuación.

Isotropía: Distribución homogénea de las partículas materiales en un sólido por la que cualquier línea o plano que se considere contendrá siempre, estadísticamente, el mismo número de partículas y de la misma naturaleza.

Anisotropia: Distribución de las partículas materiales en un cuerpo por la que cualquier línea o plano que se considere contendrá siempre, estadísticamente, distinto número o tipo de partículas.

Propiedad escalar: Propiedad física (respuesta de la materia a un estímulo físico) cuyo valor no depende de la dirección en que se mida.

Propiedad vectorial: Propiedad física cuyo valor depende de la dirección en que se mida.

Históricamente, fue previa la observación de que había propiedades cuyo valor no dependía de la dirección en que se midieran (densidad, temperaturas de cambio de estado, radiactividad más tardíamente), mientras otras sí mostraban diferencias más o menos acusadas para direcciones distintas (casi todas las restantes); así se diferenciaron las propiedades escalares de las vectoriales. AI tratar de generalizar el comportamiento implícito en cada uno de los tipos de propiedades y referirlo a la materia en sentido abstracto, se consideró ésta como «iśtropa» cuando sus propiedades no mostraban dependencia de la dirección (a lo cual alude el término, construido con las palabras griegas adecuadas), y «anisótropa», en el caso contrario; porque es evidente que nosotros sólo tenemos acceso a la naturaieza íntima de la materia a través de las respuestas que ella misma nos da a ciertos estímulos externos; y a partir de este comportamiento es como podemos esbozar explicaciones más o menos satisfactorias sobre las razones que lo justifiquen.

Pero el hecho de que la única forma de que dispongamos para reconocer la isotropía o anisotropía de la materia sea ver cómo reacciona frente a los distintos estímulos que podemos aplicarle, es decir, qué valores presentan sus propiedades, no implica que se confundan ambos tipos de conceptos. La Isotropía o Anisotropía es una cualidad inherente a la materia, relacionada con la distribución de sus partículas en el espacio; si esta distribución es al azar, efectivamente no hay razón alguna para que ninguna propiedad muestre diferencias entre direcciones oplanos distintos, porque en todos ellos seencontrará siempre el mismo tipo de partículas y el mismo número de ellas; sin embargo, si hay un cierto orden, por mínimo que éste sea, aparecerán ya direcciones o planos preferenciales para un determinado comportamiento, incluso cuando no lo podamos detectar porque los instrumentos de medida que usemos no sean capaces de apreciar intervalos tan pequeños como los que se dan en la realidad (no tengan una capacidad de resolución suficiente). Se puede recurrir para ilustrarlo a los dos ejemplos más conocidos de comportamientos de este tipo, que son la dureza y la birrefringencia; con un instrumento tan burdo y con intervalos tan desiguales como la Escala de
Mohs no podemos pretender apreciar las mínimas diferencias de dureza que se dan entre la dirección de la arista de un cuadrado y la de su diagonal, por ejemplo, a pesar de que, teóricamente, se puede predecir que las habrá, a igualdad de las demás condiciones (porque una distancia es mayor que la otra en un factor de $\sqrt{2} \mathrm{y}$, por tanto, serán diferentes las fuerzas de cohesión que mantienen unidas las partículas, fuerzas responsables de la resistencia al rayado, que es la dureza consagrada en el uso mineralógico); sólo en un caso esa diferencia entre dos direcciones (perpendiculares entre sí concretamente), es suficientemente acusada como para que la detecte esa escala (y a esa dualidad de durezas alude uno de los nombres del mineral, cianita o «distena»). Igual ocurrirá con la capacidad de dar dos rayos refractados (birrefringencia); si la diferencia de úndices de refracción para direcciones distintas es tan pequeña que los dos rayos refractados salen formando entre sí un ángulo mínimo, el ojo humano no podrá distinguirlos y no apreciará «anisotropía óptican (aunque de hecho exista, como ocurre con todos los minerales no cúbicos, salvo para una o dos direcciones de monorrefringencia); sólo en un mineral frecuente (aunque no el único), esa diferencia de velocidades de la luz es suficientemente grande como para que los dos rayos salgan tan dispares que los pueda percibir el ojo desnudo y apreciemos dos imágenes del mismo objeto si colocamos un cristal de calcita sobre él.

Así pues, parece que queda clara la diferencia conceptual entre ambas parejas; y que un mineral es anisótropo independientemente de que la dureza o la birrefringencia (u otra propiedad determinada y concreta en cada caso) muestre o no diferencias de valor según la dirección; y una propiedad es vectorial siempre que dependa, teóricamente, de la dirección, aun cuando no sea posible, con un determinado instrumento de medida, medir las diferencias entre ellas. Es evidente que en la materia isótropa (que no constituye minerales, por definición) no tiene sentido la distinción entre propiedades escalares y vectoriales (si no es por relación a la anisótropa); en ella todos Ios planos y todas las direcciones son totalmente equivalentes por estructura y composición y, por tanto, no pueden dar ninguna respuesta diferencial a ningún tipo de estímulo.

\section{ENCUESTA}

La encuesta, muy simple y anónima, trataba, escuetamente, de detectar si los alumnos conocen los conceptos implicados en el estudio, sin entrar siquiera a medir la comprensión de los mismos que puedan tener.

Con objeto de prever posibles correlaciones con ciertos factores como el tipo de centro en que se estudió o el número đe años transcurridos desde entonces, se añadían otros tres datos informativos:

- «Último curso en que estudió Mineralogía».

- «Años transcurridos» (desde entonces).

- "Tipo de centro» en que lo hizo.

- Definir Isotropía:

- Definir Anisotropía: 
- Definir Propiedad Escalar:

- Definir Propiedad Vectorial:

\section{RESULTADOS OBTENIDOS}

Las respuestas, tal como ya se esperaba por la experien" cia previa en cursos anteriores, fueron mayoritariamente erróneas y, muchas de ellas, cíclicas entre Isotropía Propiedad escalar y Anisotropía - Propiedad vectorial, como se verá en la discusión de resultados.

Para ello se recogen a continuación las respuestas obtenidas (la numeración es aleatoria, conforme se han ido encontrando, salvo para el grupo 0) y las no contestadas (B1), y separando las correspondientes a IsotropíaAnisotropía (A) de las correspondientes a las Propiedades (B), puesto que se dan combinaciones variadas entre ambas parejas. Así resultará más cómodo hacer referencia a ellas en el análisis posterior.

\section{Co) Respuestas aceptadas como correctas}

A) Iso.: «Es cuando, tomemos la dirección que tomemos, nos encontramos con igual número de elementos y en el mismo orden y distancia».

Anis.: «Es cuando presenta cierta ordenación espacial y no da igual la dirección que tomemos para ver distancias y elementos».

B) P.E.: «Aquélla que se expresa sólo con un valor», o "Aquélla que no varía, independientemente de la dirección tomada».

P.V.: «Aquélla que se expresa según los ejes del plano o espacio", 0 «Aquélla que sí varía dependiendo de la dirección tomada».

\section{Respuestas erróneas}

\section{Grupo 0}

Respuestas aberrantes:

A) Iso.: «Es la característica de los minerales que se presentan con su forma real».

Anis.: «Es la caractexística de los minerales que se presentan con una forma irreal».

B) P.E: «Es la propiedad física como son brillo, color, sabor, etc.» o «Propiedad de perpendicularidad».

P.V: «Es una propiedad física como son dureza, estructura cristalina, etc.» 0 «Posición de la roca in relación con unas coordenadas».

\section{Grupo I}

A) Iso.: «Propiedad de la materia cuyas propiedades rıo varian tomemos la dirección que tomemos".
Anis.: «Propiecad de la materia en donde las propiedades cambian según la dirección en que estudiemos dicha materia».

B) P.E: «Son aquéllas que no dependen de la dirección ni del sentido».

P.V: «Propiedades que cambian según la dirección y el sentido».

\section{Grupo 2}

A) Iso.: «Materia amorfa».

Anis.: «Materia cristalina»,

B) P.E.: «Es una magnitud que se puede representar en una escala».

P.V.: «Es una magnitud que no se puede representar en una escala».

Grupo 3

A) Iso.: "Que tiene unas propiedades concretas y bien definidas independientemente del medio en que se encuentre».

Anis.: «Aquél en que sus propiedades no son fijas sino que dependen de una serie de factores».

B) P.E.: «Aquélla que queda determinada por un número concreto".

P.V.: "Aquélla que queda determinada por un vectors.

Grupo 4

A) Iso.: «Forma de ordenación regular».

Anis.: «Forma de ordenación irregular».

B) P.E.: «Números que representan medidas de una forma exacta, sin necesidad de nada más».

P.V.: «Aquélla que posec un vector, es decir, no está definida si no se dan la dirección, módulo, sentido.»

Grupo 5

A) Iso:: «Cuando un mineral presenta iguales características en un determinado plano".

Anis.: «Lo contrario a isotropía».

\section{Grupo 6}

A) Iso.: «Es la propiedad que tienen los cuerpos en la que cuerpos diferentes poseen las mismas propiedades».

Anis.: «Es la propiedad que tienen los cuerpos en la que cuerpos iguales presentan diferentes propiedades». 
B) P.E.: «Es aquella propiedad que pođemos resaitar đe un objeto a simple vista, sin haberlo somtido a estudio».

P.V.: «Es aquella propiedad de un cuerpo, una vez. que lo hemos sometido a un proceso».

\section{ANÁLISIS DE RESULTADOS}

Los cuadros de respuestas, siguiendo la clave establecida en la clasificación anterior, resultan muy indicativos $y$ precisan poco comentario. Son los siguientes:

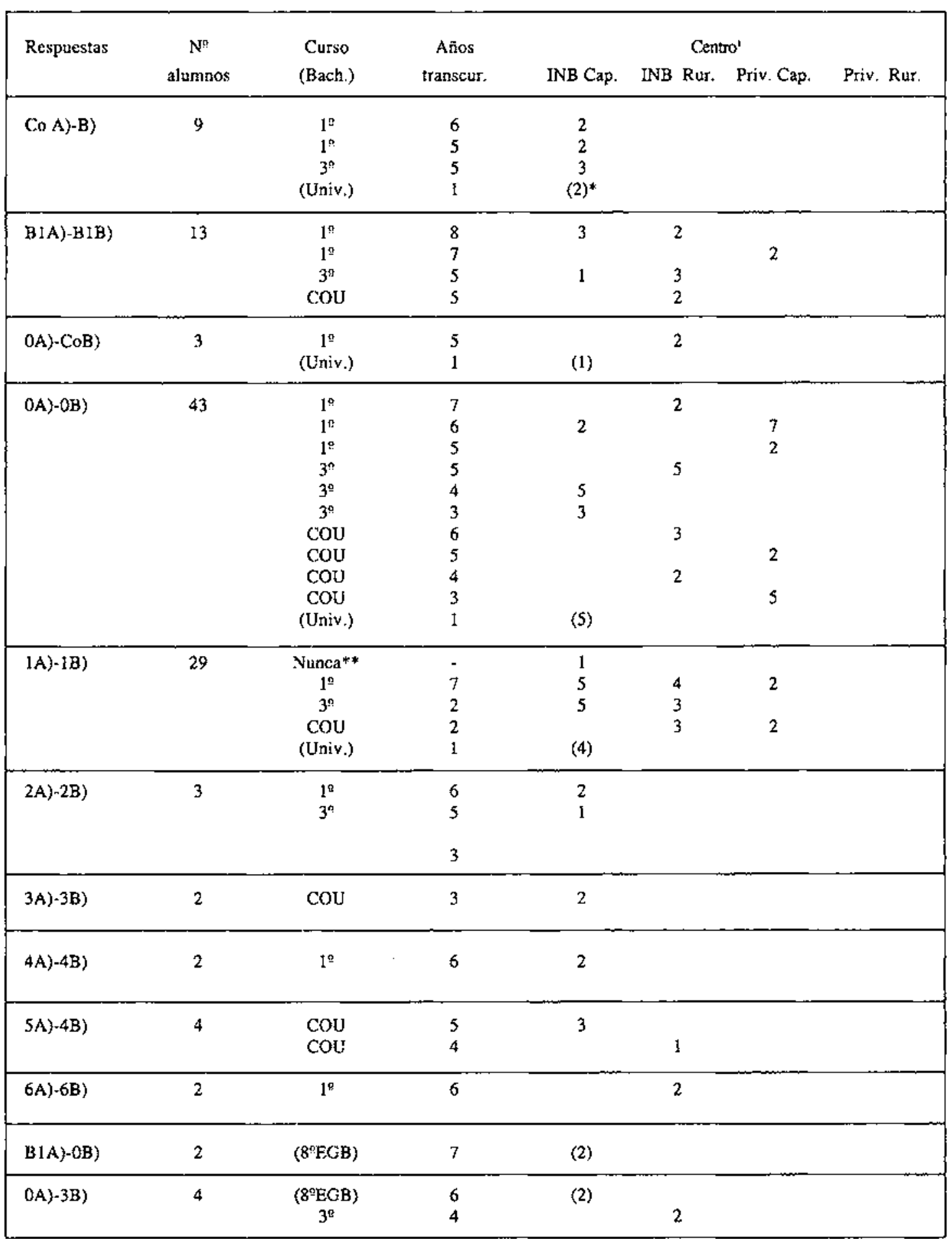

'Las cifras correspondientes a este apartado no coinciden con los totales porque algunos aiumnos no han contestado este epígrafe.

* Estos datos se presentan entre paréntesis para jndicar el númeso de alumnos en estas condiciones, pero sin que se puedan asignar a un centro concreto de los reseñados. 
(Continuación)

\begin{tabular}{|c|c|c|c|c|c|c|c|}
\hline \multirow[t]{2}{*}{ Respuestas } & \multirow{2}{*}{$\begin{array}{c}\mathrm{N}^{\circ} \\
\text { alumnos }\end{array}$} & \multirow{2}{*}{$\begin{array}{l}\text { Curso } \\
\text { (Bach.) }\end{array}$} & \multirow{2}{*}{$\begin{array}{c}\text { Años } \\
\text { transcur. }\end{array}$} & \multicolumn{4}{|c|}{ Centro' } \\
\hline & & & & INB Cap. & INB Rur. & Priv . Cap. & Priv. Rur. \\
\hline \multirow[t]{11}{*}{0 A) $-4 B)$} & 71 & (8. EGB $)$ & 7 & (1) & & & \\
\hline & & I음 & 9 & & 2 & & \\
\hline & & I은 & 8 & 2 & 2 & & \\
\hline & & $I^{2}$ & 7 & & & 2 & \\
\hline & & $i^{2}$ & 6 & 8 & 9 & 3 & \\
\hline & & $3^{a}$ & 6 & 4 & & & \\
\hline & & $3^{\circ}$ & 5 & 5 & 2 & & \\
\hline & & $3^{9}$ & 4 & 5 & 5 & & \\
\hline & & $3^{9}$ & 3 & 4 & 3 & 1 & \\
\hline & & $\mathrm{COU}$ & 5 & 5 & & & \\
\hline & & $\mathrm{COU}$ & 3 & & 5 & 3 & \\
\hline \multirow[t]{2}{*}{ (A)-3B) } & 5 & $\mathrm{COU}$ & 5 & 3 & & & \\
\hline & & $\mathrm{COU}$ & 4 & & 2 & & \\
\hline \multirow[t]{2}{*}{ (A)-4B) } & 4 & $\mathrm{COU}$ & 4 & 2 & & & \\
\hline & & $\mathrm{COU}$ & 3 & & 2 & & \\
\hline \multirow[t]{2}{*}{ (A) $-6 \mathrm{~B})$} & 2 & $1^{0}$ & 7 & & 1 & & \\
\hline & & $1^{8}$ & 6 & & 1 & & \\
\hline Total & 198 & & & & & & \\
\hline
\end{tabular}

** Esta respuesta indica que el alumno no estudió ni se examinó de esta parte del programa de I̊ de Bach (obligatorio), y lo que sabe lo habrá aprendido por su cuenta.

Como se ve, sólo el 5\% contesta correctamente (inchuso con el margen de tolerancia expresado); un $6 \%$ no sabe y no contesta; y un $2 \%$ contesta correctamente sólo lo referente a las propiedades (de los cuales un $1 \%$ es repetidor de curso en la Universidad).

El resto, un $87 \%$, aproximadamente, contesta de forma totalmente incorrecta o incluso aberrante (un $21 \%$ ) a ambos tipos de conceptos. Entre ellos, un grupo significativo (un 20\%) confunden los conceptos entre sí y los definen de forma claramente parecida. Si consideramos suficientemente similares $\mathrm{O}$, al menos, indicadores de la misma concepción de fondo la $5 \mathrm{~A}$ con la $1 \mathrm{~A}, \mathrm{y}$ las $2 \mathrm{~B}$, $3 \mathrm{~B}$ y $4 \mathrm{~B}$ con Ia $\mathrm{IB}$, podría deducirse que, efectivamente, se da una confusión total entre ambos tipos de conceptos en un porcentaje muy alto de alumnos (del orden del $63 \%$ ).

Se hace necesario buscar las causas de esta situación.

\section{HIPÓTESIS EXPLICATIVAS}

\section{Presentación defectuosa por parte del profesor}

Evidentemente, ésta es una posibilidad a tener en consideración, desde un punto de vista teórico, vistos los resultados. Para contestar adecuadamente a ella sería necesario hacer una encuesta similar a la anterior entre los profesores, inciuidos los de último Ciclo de EGB por si ya introdujeron en su programa estos contenidos (como se detecta, en algunos casos). No era la finalidad del trabajo $y$, por lo tanto, esta hipótesis queda sin contestación por el momento.

No obstante, quizá el análisis de los libros de texto (escritos por profesores similares a los que imparten las clases) pueda permitir inclinarse en algún sentido (y orientar en él la investigación); los resultados que se obtengan de ellos pueden tomarse como indicio estimativo de probabilidad de la certeza o falsedad de esta hipótesis.

\section{Presentación defectuosa en la bibliografía}

Conociendo por propia experiencia el peso que tiene «to escrito» en los alumnos, que tienden a aceptarlo acríticamente (incluso en los niveles universitarios, lamentablemente), era obligado considerar la hipótesis de que Ios errores vinieran condicionados por la bibliografía usada en el estudio de los cursos en que se imparte Geología (con Mineralogía). Por ello he recopilado los textos más usuales de los últimos años (pertenecientes todos al Plan de Estudios de 1975, el último antes de Ia Reforma que está a punto de implantarse con carácter general) en los centros de Bachillerato, y he analizado sus contenidos en 
lo que a los conceptos en litigio se refiere. Aunque ya los cuestionarios oficiales vigentes sugieren que sólo los de primer curso podrían tratar estos conceptos, he comprobado que, efectivamente, es así; los de tercero se enfocan desde un punto de vista más dinámico y genético y no tratan las características de la materia mineral, suponiéndolas conocidas ya de primero.

Los resultados son los siguientes, presentados por orden alfabético de editoriales:

ANAYA, 1981, p. 21: «Iguamente, la materia cirstalina es anisótropa, es decir, que según la dirección del espacio que se considere, las distancias que separan a dos átomos o moleculas sucesivos varian; esto afecta a muchas de las propiedades físicas del mineral».

(No he encontrado la definición de propiedades escatares y vectoriales)

BRUÑO, 1975, p. 33: «Isótropo: las propiedades se manifiestan por igual en todas direcciones. Anisótropo: Ias propiedades varian con la dirección y dependen de ella».

"Las propiedades físicas pueden ser escalares y vectoriales.

«Escalares son las que se representan por un solo dato, independiente de la dirección y la intensidad. Las propiedades vectoriales, en cambio, necesitan intensidad, direccion y sentido para ser representadas».

DIDASCALIA, 1981. p. 38: «El hecho de que varíe la longitud de la traslación desde un nudo a cada uno de sus vecinos hace que el valor de las propiedades vectoriales varíe con la dirección: Anisotropían.

(No he encontrado la definición de propiedades escalares y vectoriales).

ECIR, 1979, pp. 47 y 49: «Se llaman cuerpos isótropos aquéllos en los que una misma propiedad física se manifiesta con igual intensidad en todas las direcciones. Son anisótropos cuando estas mismas propiedades físicas cambian de intensidad según las direcciones consideradas»; (4 líneas después dice que «Ia anisotropía es un carácter propio de la materia cristalina, siendo consecuencia de la ordenación de sus efementos integrantes según redes paralelepipédicas»).

«Las propiedades físicas que cambian con la dirección en los cuerpos minerales se denominan propiedades vectoriales, y las que no dependen de la dirección, escalaresy.

(Nota: Fn la nueva ediciŏn de 1987 , parecen haberse eliminado todas las alusiones a estos conceptos, porque no los he encontrado).

EDELVIVES, 1985: (No he encontrado las definiciones en cuestión).

\section{REFERENCIAS BIBLIOGRÁFICAS}

AMORÓS, J.L., 1978. El cristal. (Urania: Barcelona).

DíAZ-MAURIÑO, C., 1988. Prácticas de Mineralogía. (Alhambra: Madrid).

GALLEGOS, J.A., 1977. Claves Mineralógicas, Granada.

HOCHLEITNER, R., 1983. Minerales y Rocas. (Omega: Barcelona).
SANTILLANA, 1985: No he encontrado ninguna de las definiciones en cuestión.

SM, 1986: No he encontrado ninguna de las definiciones en cuestión.

VICENS-VIVES, 1980 , p. 31 (recuadro adicional lateral): «Se dice que un cristal es anisótropo para una propiedad cualquiera (refringencia, dureza, etc.) cuando el valor de esta propiedad varía según la orientación en la que se realice el ensayo. Si no existen variaciones relacionadas a diferentes orientaciones el mineral es isotropo."

( No he encontrado las definiciones de propiedades escalar y vectorial).

\section{CONCLUSIONES}

A partir de los resultados obtenidos en la encuesta y la similitud con Ios enunciados existentes en los libros de texto consultados (de los que sólo el de la editorial Anaya los define correctamente), parece autorizado concluir:

1. Los errores de concepto que se detectan en los alumnos para los conocimientos examinados son consecuencia del defectuoso proceso de enseñanza que se les ha proporcionado.

2. La influencia de los libros de texto en el aprendizaje a niveles de Bachillerato es casi total.

3. Muy probablemente, la enseñanza impartida por los profesores no ha corregido la defectuosa presentación de esos conceptos realizada por los libros de texto.

4. Muy probablemente, los profesores se limitan a programar el desarrollo de la asignatura siguiendo, sin más, los libros de texto que han clegido.

5. Muy probablemente, los profesores no se han tomado la molestia de corregir los libros de texto en estos contenidos.

6. Probablemente, los profesores no inculcan en sus alumnos la necesidad de utilizar el libro de tex to como una fuente bibliográfica más de consulta que hay que analizar críticamente.

7. Podría ser interesante completar la investigación indagando si hay relaciones, y cuáles, entre un determinado conocimiento de estos conceptos y los libros de texto usados en el curso correspondiente a su aprendizaje.
HURBULT, C.S., 1982. Manual de Mineralogía. (Reverté: Barcelona).

KLOOKMANN, A., 1964. Tratado de Mineralogia. (Gustavo Gili: Barcelona).

PHILIPS, W.J. y N., 1982. Fundamentos de Mineralogía para geólogos. (García Peña: Madrid). 